\section{Antología del pensamiento crítico caribeño contemporáneo (West Indies, Antillas Francesas y Antillas Holandesas), Félix Valdés García (coord.) CLACSO, Buenos Aires 2017, pp. 608, ISBN 978-987-722-253-11}

Anuario Latinoamericano Ciencias Políticas

y Relaciones Internacionales vol. 6, 2018

pp. 289-293

DOI: 10.17951/al.2018.6.289-293

\section{Anthology of Contemporary Caribbean Criticism (West Indies, French Antilles and Netherlands Antilles), Félix \begin{abstract}
Valdés García (coord.)
CLACSO, Buenos Aires 2017, pp. 608,
ISBN 978-987-722-253-1

CLACSO, Buenos Aires 2017, pp. 608,
ISBN 978-987-722-253-1
\end{abstract}

El Caribe se constituye en un inmenso mosaico multidimensional (cultural, político, intelectual, etc.) marcado por una amplia diversidad de dominaciones, colonizaciones, influencias, resistencias y reelaboraciones, que están explicitadas en sus diversas denominaciones. De esta forma, una parte de la región, el Caribe hispano, se llama "El Caribe", en las islas anglófonas se denomina "West Indies" o "The Caribbean", en la zona francesa se señala como "Les Antilles" o "La Caraïbe", el área holandesa se denomina "Dansk Vestindien". Estas diferentes denominaciones demuestran el carácter uno y diverso de la región, combinados dialécticamente, que explicitan, de una u otra forma, toda su diversidad y potencialidad y esa obra trata de la producción del pensamiento crítico en las tres últimas.

En realidad, estas áreas del Caribe, a pesar de compartir con Iberoamérica una historia común, que antecede a la colonización europea, y de los lazos históricos, económicos y culturales desarrollados desde hace siglos, el pensamiento crítico caribeño sigue ignorado o marcado (todavía) por el exotismo, ya que la centralidad intelectual de los países del norte, principalmente en su vertiente anglosajona, sigue condicionando nuestra mirada y nuestra reflexión sobre una región tan cercana y, al mismo tiempo, tan distante.

En este sentido, la obra se constituye en un trabajo fundamental y muy instigador para el (re)conocimiento del pensamiento crítico caribeño y sus lazos con la realidad iberoamericana, principalmente de las poblaciones originarias

1 Disponible en: http://biblioteca.clacso.edu.ar/clacso/se/20170707025855/AntologiaDePensamientoCriticoCaribeno.pdf 
y de los afrodescendientes, y la construcción de alternativas para una sociedad más justa y equitativa.

La obra es parte integrante de la serie Países de la colección de Antologías del Pensamiento Social Latinoamericano y Caribeño, publicada por el Consejo Latinoamericano de Ciencias Sociales (CLACSO) ${ }^{2}$, en la que ya se ha divulgado la producción crítica contemporánea de Uruguay, Panamá, El Salvador, Nicaragua, Argentina, Bolivia, Chile, Colombia, Cuba, México, la República Dominicana, Perú, Paraguay y Venezuela, y próximamente nuevos volúmenes sobre otros países deben ser lanzados.

La colección se compone de cinco series: Trayectorias, Países, Pensamientos Silenciados, Miradas Lejanas y CLACSO/SIGLO XXI (publicación conjunta), cuyos textos pueden ser considerados esenciales para conocer y comprender el pensamiento social latinoamericano y caribeño, clásico y contemporáneo.

Este trabajo fue organizado por el profesor cubano Félix Valdés García, miembro de la Cátedra sobre el Caribe de la Universidad de La Habana, de la Asociación de Estudios del Caribe (AEC) y del Consejo Latinoamericano de Ciencias Sociales (CLACSO).

La obra, como la región retratada, está marcada por la diversidad de temáticas y perspectivas y se inserta en la dinámica de la colección, buscando retratar una amplia gama de autores y enfoques que poseen como hilo conductor un pensamiento crítico, basado en las condiciones y desafíos que la región enfrenta desde la colonización, presentando autores clásicos del pensamiento caribeño como Frantz Fannon, Aimé Césaire, Eric Willians, Cyril Lionel Robert James, Edward Kamau Brathwaite y Maurice Bishop, así como autores contemporáneos como Sir William Arthur Lewis, Kari Polanyi Levitt, Lloyd A. Best, Sylvia Wynter, Jean Bernabé, Patrick Chamoiseau y Raphaël Confiant.

De esta forma, el libro adquiere una coherencia y una relativa unidad, incluso en la diversidad, pues los autores presentados logran demostrar la profunda relación entre sus análisis y la dinámica social, política y económica de sus países y de la región, produciendo un pensamiento que busca contribuir a la transformación social, en sus diversas formas y movimientos, superando los colonialismos, en todas sus manifestaciones y actualizaciones.

Además, el trabajo está marcado por un enfoque multidisciplinario y por el diálogo fructífero de un conjunto de corrientes relacionadas con el pensamiento crítico, en el que se destacan tres tradiciones. En primer lugar, el pensamiento marxista, utilizado de forma creativa y distanciada de dogmatismo y esquematismo, asociado al marxismo occidental y, principalmente, al impulso renovador y originario iniciado por la Revolución Cubana y por las

2 El Consejo Latinoamericano de Ciencias Sociales (CLACSO) es una institución no gubernamental, creada en 1967 y asociada a la UNESCO, que reúne cerca de 394 centros de investigación, programas de postgrado o instituciones en ciencias humanas y sociales de 26 países de América Latina. Además de esto, también están afiliadas diversas instituciones de EE. UU., Europa, África y Asia que se dedican al estudio de temas latinoamericanos. Para conocer la entidad se puede acceder a la página: www.clacso.org.ar 
relecturas marxistas desarrolladas en África y América Latina a lo largo de las últimas décadas. Además de él, se destaca el pensamiento negro, originario de la diáspora africana, y su relectura de la colonización y de la esclavitud, así como de la condición de los negros en el mundo contemporáneo, buscando rescatar sus raíces, originalidad y relevancia para la superación de la condición subalterna y subdesarrollada.

Por último, emergen los trabajos que, manteniendo un diálogo con las perspectivas anteriores, incorporan las contribuciones del pensamiento decolonial, anticolonial o postcolonial, buscando deconstruir la modernidad eurocéntrica, sus lógicas y determinaciones, rescatando los diversos elementos (culturales, políticos, etc.) asociados a la negritud, las poblaciones originarias y las mujeres, entre otras, que nos permiten repensar la condición colonial y, principalmente, la dependencia y el desarrollo económico y social de la región.

En este sentido, podemos señalar que la obra puede ser analizada a partir de cinco ejes fundamentales, que se entrecruzan y se desdoblan en innumerables reflexiones.

El primer eje, que atraviesa toda la obra, está asociado a la temática del colonialismo, tanto en su dimensión temporal como en los efectos políticos, económicos y culturales, al promover un cuadro de dominación económica, política y cultural, asociado a la colonialidad del saber y del poder en esta región y en toda América Latina. En este sentido, se destacan los análisis, que pueden ser considerados clásicos, de Frantz Fanon (Los condenados de la tierra - fragmentos) y de Aimé Césaire (Discurso sobre el colonialismo) y el trabajo, más reciente, de Sylvia Wynter (1492: Una nueva visión del mundo), aunque los demás textos del libro puedan ser considerados un desdoblamiento de este eje primordial y continúen, a su modo, discutiendo tal temática.

El segundo eje se caracteriza por el debate sobre la dependencia y la condición periférica de los Estados caribeños, además de los desafíos para la creación de una dinámica efectiva de desarrollo económico y social. En este sentido, se presentan los trabajos de Eric Williams (Capitalismo y esclavitud y El futuro del Caribe), Kari Polanyi Levitt y Lloyd A. Best (Un enfoque histórico e institucional del desarrollo económico caribeño y Bosquejo de una teoría general de la economía del Caribe y Sir William Arthur Lewis (La agonía de las ocho, texto extraído de Teoría para el desarrollo económico y social del Caribe), entre otros.

El tercer eje, presente en buena parte de los trabajos, está relacionado con la cuestión de la negritud y la condición "criolla", discutidas en múltiples dimensiones, en las que se destacan los elementos culturales y políticos. En el primer caso, están presentes trabajos que revisan el tema de la esclavitud, en su implicación social y cultural, analizan la emergencia de la noción de negritud, en su relación con el pan-africanismo, y, más recientemente, de la noción de "creolidad", en que se destacan los trabajos de Edward Kamau Brathwaite (La criollización en las Antillas de lengua inglesa), de George Lamming (Los placeres del exilio) y de Jean Bernabé, Patrick Chamoiseau y Raphaël Confiant (Elogio de la creolidad - fragmentos).
Anuario Latinoamericano Ciencias Políticas y Relaciones Internacionales vol. 6, 2018

pp. 289-293

DOI: 10.17951/al.2018.6.289-293 
En el plano político y social, se destacan trabajos que discuten la emergencia del movimiento negro y de una conciencia y movilización social regional como los textos de Édouard Glissant (El discurso antillano), de Walter Rodney (El Black Power. Su relevancia en el Caribe) o de Brian Meeks (Radical Caribbean: From Black Power to Abu Baker /Caribe radical: del Black Power a Abu $B a k r)$, entre otros.

El cuarto eje se relaciona con el impacto de las revoluciones, pasadas y presentes, en la constitución y desarrollo de un pensamiento revolucionario y libertario, que incorpora las tradiciones culturales, africanas y amerindias presentes en la región, y busca desarrollar un proyecto político-cultural que combine justicia social y valorización de estas tradiciones subalternizadas a lo largo de la historia caribeña. En este sentido, diversos trabajos promueven un contacto y un diálogo con el impulso libertario de la Revolución Cubana y de los procesos de descolonización e independencia en África, a partir de los años 60, de los cuales podemos destacar los textos de Cyril Lionel Robert James (De Toussaint L'Overture a Fidel Castro, extraído de Los jacobinos negros), de Maurice Bishop (jSiempre adelante! Contra el imperialismo y hacia la independencia nacional legítima y el poder del pueblo) y de Lloyd A. Best (Pensamiento independiente y libertad caribeña), entre otros.

El último eje se relaciona con temáticas emergentes, entre las cuales se destacan las cuestiones culturales y de género que buscan, a partir de la condición caribeña, redefinir el papel y la organización de estas sociedades y, en el segundo caso, revisar la condición subalterna de las mujeres en el mundo occidental y contribuir a la superación de esta condición y del empoderamiento femenino. En este sentido, se destacan los trabajos de Elsa Goveia (Estudio de la historiografía de las Antillas inglesas hasta finales del siglo XIX), de Terry Agerkop (Las culturas tradicionales y la identidad cultural en Surinam) y, principalmente, de Alissa Trotz (Género, generación y memorias: tener presente un Caribe futuro).

Además de los aspectos ya mencionados, otros elementos emergen de la lectura de esta obra. Como demuestran los textos, los diversos autores logran captar con acierto la dicotomía entre la unidad y la diversidad que caracterizan al Caribe, principalmente, al destacar sus diversas tradiciones, pero que convergen hacia una historia y destinos comunes, además de revelar la dinámica política y social de las pequeñas naciones. Además, demuestran que tales autores buscan asociar compromiso y sensibilidad social con rigor intelectual, haciéndose relevantes para el desarrollo de un pensamiento propio, caribeño y latinoamericano, fundamentado tanto en la realidad particular de cada isla como en los desafíos comunes que marcan la región y, de cierta forma, toda América Latina.

Sin embargo, como toda colección, resultado de opciones del organizador y de los límites de la publicación, aunque posee innumerables méritos y se trata de un trabajo muy importante, tiene limitaciones relacionadas, principalmente, a la ausencia de algunos pensadores y de algunas temáticas, como 
el análisis de las instituciones y de la dinámica contemporánea de la integración regional caribeña, las relaciones recientes con la herencia y el continente africano, el agravamiento de problemas sociales y ambientales, la aparición de nuevas formas de organización cultural y política y el papel de las nuevas generaciones en la construcción de un marco del pensamiento crítico caribeño, entre otras.

A pesar de esto, es posible apuntar que la obra, así como toda la colección de CLACSO, al presentar las reflexiones fundamentales del pensamiento crítico latinoamericano contemporáneo, es esencial para el conocimiento de América Latina y el Caribe, en su unidad y diversidad, pues como señala el compilador Félix Valdés García: "Así, más allá de la obra de Jamaica, Trinidad y Tobago, Martinica o Barbados, los textos reunidos expresan el cuestionamiento de una totalidad mayor, dada con mayor frecuencia en las lenguas de Próspero que en creole, papiamento o sranang tongo, pero tan agudo como la plaga roja que Caliban pronunciara a Próspero. Ella expresa la unidad de lo diverso, de lo individual y lo universal de una experiencia, de islas que se repiten una y otra vez, sin ser iguales ni siquiera consigo mismas, más allá de la proximidad física, la fragilidad, la continuidad fáctica que la historia puso a merced de antojos imperiales y de experimentos sociales y culturales más impensados de la civilización occidental. Sirva la presente selección para rebasar las divisiones, el desconocimiento de pueblos que comparten una misma suerte, semejantes herencias y un mismo sol insular y de Nuestra América" (p. 34). ¡A todos, buena lectura!

Marcos Antonio da Silva Universidade Federal da Grande Dourados, Brasil MarcosSilva@ufgd.edu.br https://orcid.org/0000-0003-1196-2814
Anuario Latinoamericano Ciencias Políticas y Relaciones Internacionales vol. 6, 2018

pp. 289-293

DOI: 10.17951/al.2018.6.289-293 